\title{
Diffraction efficiency of a double-pass acousto-optic modulation system
}

\section{Eficiencia de la difracción de un sistema de modulación acusto-óptico de doble paso}

\author{
J. Barrios ${ }^{1 *}$, J. Álvarez ${ }^{1}$, F. Racedo N. ${ }^{1}$. \\ 1. Grupo de Espectroscopía Óptica y Emisión Láser, Universidad del Atlántico, Puerto Colombia, Colombia \\ ${ }^{*}$ E)-mail: franciscoracedo@mail.unitlantico.edu.co S: miembro de SEDOPTICA / SEDOPTICA member \\ Received: $18 / 10 / 2020 \quad$ Accepted: $18 / 01 / 2021$ \\ DOI: 10.7149/OPA.54.1.51051
}

\begin{abstract}
:
We described the characterization of an acousto-optic modulation system and the optimization of the set up to improve the efficiency of diffraction in single and double-pass configurations. In the singlepass configuration, the system consists of an acousto-optic modulator, a voltage-controlled oscillator (VCO) and a radio frequency amplifier, in which the diffraction efficiency and the optical power of the diffracted beam were characterized in the function of the applied radiofrequency. Additionally, taking as a reference the need to correct the variation of the diffraction angle that may occur when the system is modulated in frequency in a simple step, its correction is presented by means of the implementation of the configuration in double step. The double-pass configuration consists of a VCO that controls the incoming acoustic signal to the modulator, a modulator which diffracts the incident light and this passes through a retarder sheet to avoid the retroreflection of the beam; Then, using an iris, only order 1 of diffraction of the beam was allowed to pass and a plane mirror was used for working in the infrared which directs the laser in the second pass of it in the modulator. Once the double-pass system had been optimized, the diffraction efficiency curve was obtained. This work is presented as an updated guide for the implementation of an acousto-optic modulation system in a double-pass configuration.
\end{abstract}

Key words: Acousto-optic modulation, single pass, double pass, AOM optimization.

\section{RESUMEN:}

Se describe la caracterización de un sistema de modulación acusto-óptico y la optimización del set up para mejorar la eficiencia de la difracción en configuraciones de paso simple y doble. En la configuración de paso simple, el sistema consta de un modulador acusto-óptico, un oscilador controlado por voltaje (V.C.O) y un amplificador de radiofrecuencia, en la cual, se caracterizó la eficiencia de la difracción y la potencia óptica del haz difractado en función de la radiofrecuencia aplicada. Adicionalmente, tomando como referencia la necesidad de corregir la variación del ángulo de difracción que se puede tener cuando se modula en frecuencia el sistema en paso simple, se presenta su corrección por medio de la implementación de la configuración en paso doble. La configuración de paso doble, consta de un VCO que controla la señal acústica entrante al modulador, un modulador el cual difracta la luz incidente y esta pasa por una lámina retardadora para evitar la retro reflexión del haz; después usando un iris se deja pasar solo el orden 1 de difracción luego se usa un espejo plano el cual direcciona el láser en la segunda pasada de éste en el modulador. Una vez optimizado el sistema en paso doble se procedió a obtener la curva de eficiencia de difracción y potencia en función de la frecuencia. Este trabajo se presenta como una herramienta actualizada para la caracterización e implementación de un sistema de modulación acusto-óptico en configuración de doble paso.

Palabras clave: Modulación Acusto-óptica, paso simple, paso doble, optimización AOM. 


\section{REFERENCES AND LINKS / REFERENCIAS Y ENLACES}

[1] A. Yariv,Quantum Electronics., Wiley (1989).

[] Donley E A, Heavner T P, Levi F, Tataw M and Jefferts S R 2005 Review of Scientific Instruments 76(6)063112 1-6

[]] Brillouin L 1922 Ann. Phys 17(2) 88-122

[4] Debye P and Sears F 1932 Proceedings of the National Academy of Sciences 18(6) 409-414

[]ㅡ Lucas R and Biquard P 1932 Journal de Physique et le Radium 3 464-477

[]]B. N. J and L. J. N,Acousto-optic signal processing: Theory and implementation, MarcelDekker, Inc. (1983)

[7]Waluyo T B, Mulyanto I and On H 2008 Jurnal Fisika Himpunan Fisika Indonesia 8(1) 30-38

[8] Korpel A 1981 Proceedings of the IEEE 69(1) 48-53

[9] Berg N J and Lee J N 1983 Acousto-optic signal processing: Theory and implementation (Optical engineering vol 2)

(New York: Marcel Dekker, Inc.)

[10] IntraAction C 2019 Model atm series acousto-optic modulator URL

[11] D. W. M Bass, E Van Stryland and W. Wolfe,Handbook of optics, McGRAW-HIL, Inc.)(1995).

[12] Jodlowski L 2003 Opto-Electronics Review 11(1) 55-64

[13] Chih-Hao Chang, R. K. Heilmann, M. L. Schattenburg, and P. Glenn Review of Scientific Instruments 79, 033104 (2008)

[14] J. M. Chowning, “The synthesis of complex audio spectra by means of frequency modula-tion,"Journal of the audio engineering society 21(7), 526-534 (1973).

[15] A. D. R. R. M Aldous, J Woods and M. Himsworth, "Carrier frequency modulation of an acousto-optic modulator for laser stabilization," (2017)

[16] S.-L. Jang, Y.-K. Wu, C.-C. Liu,et al., "A dual-band cmos voltage-controlled oscillator im-plemented with dualresonance lc tank,"IEEE microwave and wireless components letters19(12), 816-818 (2009).

\section{Introducción}

Los moduladores acústico-ópticos (AOM) son dispositivos útiles que permiten modular la dirección espacial, la intensidad y la frecuencia de un haz láser. Dentro de estos dispositivos el haz incidente se difracta en un cristal bajo la influencia de ondas acústicas que viajan a través de éste. Al variar la frecuencia y la amplitud de las ondas acústicas que se propagan por el cristal se obtiene la modulación del haz [1]. El ángulo de difracción del haz láser al pasar por el AOM cambia cuando varía la frecuencia de las ondas acústicas, en ciertas aplicaciones este cambio del haz es un efecto secundario no deseado y puede eliminarse de forma eficiente con el uso del AOM en la configuración de doble paso [2].

Se describe la caracterización de un sistema de modulación acusto-óptica para aplicación en la región del infrarrojo en configuración de paso simple y doble. Para la caracterización del sistema de modulación se optimizó el rendimiento del AOM teniendo en cuenta el ángulo y posición de entrada del haz. Una vez alineado y optimizado el sistema, se estudió la respuesta del haz de salida frente a las variaciones de frecuencia e intensidad de la señal de radiofrecuencia aplicada al cristal del AOM, seguido se obtuvo las curvas de eficiencia de la difracción en paso simple y posteriormente para la configuración en paso doble. Finalmente se discute la configuración del sistema optimizado.

\section{Teoría}

En 1922 Brillouin desarrolló la idea de dispersión de la luz usando ondas acústicas en un material [3]. Una década después Debye y Sears [4] realizaron los primeros experimentos de la teoría descrita por Brillouin y tras la invención de los láseres se incentivó la aplicación de modulación acusto-óptica, ya que puede utilizarse para el control preciso de un haz láser [5].

En una buena aproximación, el proceso de modulación acusto-óptico puede describirse como la difracción de un haz de luz en una rejilla inducida por un índice de refracción espacialmente modulado. En la Figura 1, se observa la ilustración del fenómeno, en la cual, se crean ondas acústicas (producidas por un transductor piezoeléctrico en la base del cristal) que se mueven dentro del cristal e inducen los cambios en el índice de refracción produciendo la difracción del haz [6]. 


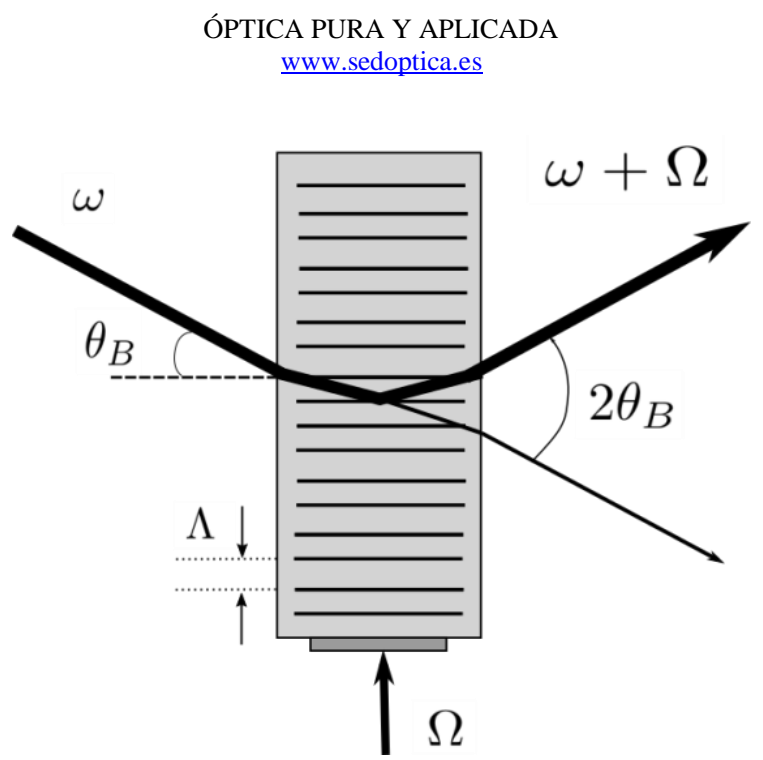

Fig.1. Esquema de la luz dispersada cuando pasa a través de un medio acusto-óptico y una frecuencia $\Omega$.

En la Fig. 1 se muestran los elementos esenciales en la modulación de la luz en un AOM como un proceso de dispersión. La condición de Bragg se puede derivar tratando las ondas acústicas y de luz como partículas con vectores de onda $\kappa_{s} \mathrm{y}$ $\kappa_{L}$ definidos a partir de la frecuencia de la onda acústica $(\Omega)$ y de la frecuencia del haz de luz incidente $(\omega)$. La absorción y emisión de fotones acústicos resultan del proceso de dispersión entre fotones y fotones, el cual cual puede ser descrito por las relaciones de energía-momento $\omega_{D}=\omega_{I}+\Omega$ y $\kappa_{D}=\kappa_{I}+\Omega$ [7].

Despreciando las variaciones de frecuencia dentro del cristal se puede encontrar la relación para el ángulo de difracción,

$$
\sin (\theta)=\frac{\kappa}{2 \kappa_{I}}
$$

La Ecuación 1 juega un rol importante, dado que cuando se satisface la relación que ésta establece, la eficiencia de la difracción del haz de primer orden en un AOM se maximiza [8].

\section{Configuración del Set-up}

Para el uso de moduladores acusto-ópticos es necesario tener un sistema de control de la señal de radiofrecuencia que proporciona la onda acústica dentro del AOM, este controlador está compuesto por tres componentes esenciales, un oscilador controlado por voltaje (VCO), un atenuador de voltaje variable (VVA) y un amplificador de radiofrecuencia [9].

Es necesario proteger el amplificador cuando se conecta un AOM a una señal de RF, el orden en que se conecten los tres componentes es crucial. Inicialmente, se debe conectar la carga de salida (AOM), luego se debe aplicar el voltaje de suministro de DC y finalmente, se debe aplicar la entrada de RF (voltajes de suministro y control a VCO y VVA). Para desconectar de forma segura un AOM de una señal de RF este orden de conexión se invierte, eliminar la entrada de RF, eliminar la tensión de alimentación DC y luego eliminar la carga de salida (AOM) [9].

A continuación se describe el set up para las configuraciones en paso simple y paso doble, además se discuten las curvas obtenidas de caracterización de la eficiencia de la difracción del haz de primer orden y potencia óptica como función de la señal de radiofrecuencia entrante.

\section{2.a. Configuración de Paso simple}

El AOM responde con la variación de la amplitud y frecuencia de la onda acústica entrante. En la Fig.2, se observa la configuración experimental utilizada. 


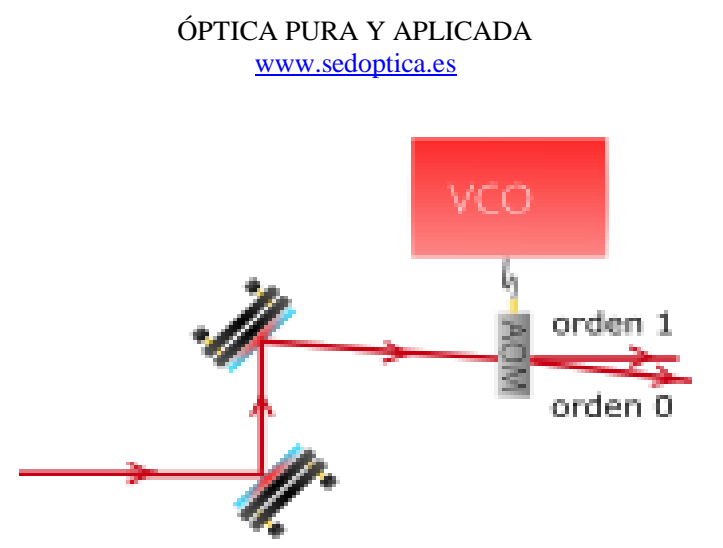

Fig.2. Set-up Experimental para configuración en pasaje simple

Como se observa en la Fig. 2, el set-up experimental consta de un driver RF (VCO) para controlar la frecuencia y amplitud de la onda acústica que se propaga a través del cristal, del mismo modo actúa como una red de difracción frente a la incidencia de la luz, cambiando el ángulo de difracción del haz. El VCO utilizado consta de un POS +150 de Mini-circuit.

Para obtener la mejor relación de dispersión del haz difractado y de esta manera maximizar la eficiencia del haz a primer orden, se varió la frecuencia, modulando entre los 70 y $150 \mathrm{MHz}$ para observar la región de mayor eficiencia de la difracción, lo cual se obtiene al revisar la relación entre la potencia óptica de entrada y salida. Teniendo en cuenta el procedimiento anteriormente mencionado en la Fig.3, se puede ver que la región donde la eficiencia de difracción es máxima se encuentra en las cercanías de los $100 \mathrm{MHz}$ obteniendo una eficiencia del 67\%, en otros términos, la eficiencia aumenta a medida que se trabaja en las cercanía de la frecuencia central del modulador y asimismo disminuye en cuando se tienen frecuencias mayores a la frecuencia central. En la Fig. 4, se observa la relación entre la potencia óptica y la amplitud modulada (Radiofrecuencia). Se moduló entre los -10 dB y $2 \mathrm{~dB}$, encontrando una relación creciente entre la potencia óptica y la frecuencia RF, este aumento se tendrá hasta que se obtenga la potencia óptica de saturación del modulador.

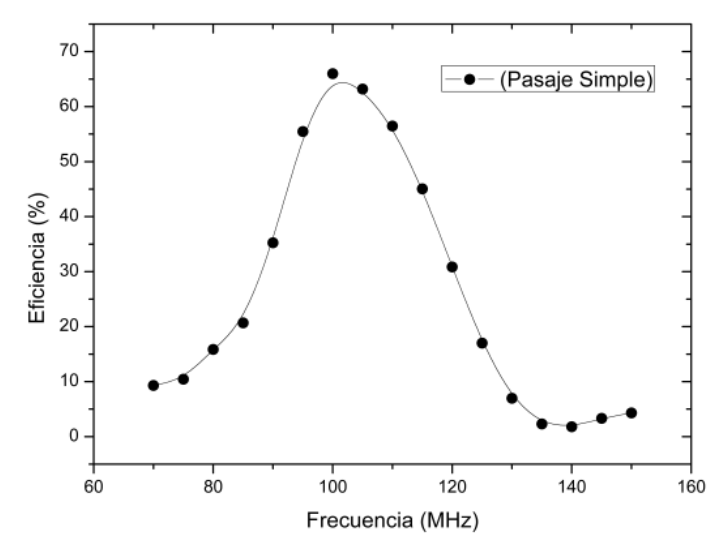

Fig.3. Eficiencia de la difracción como función de la frecuencia RF en pasaje simple. 


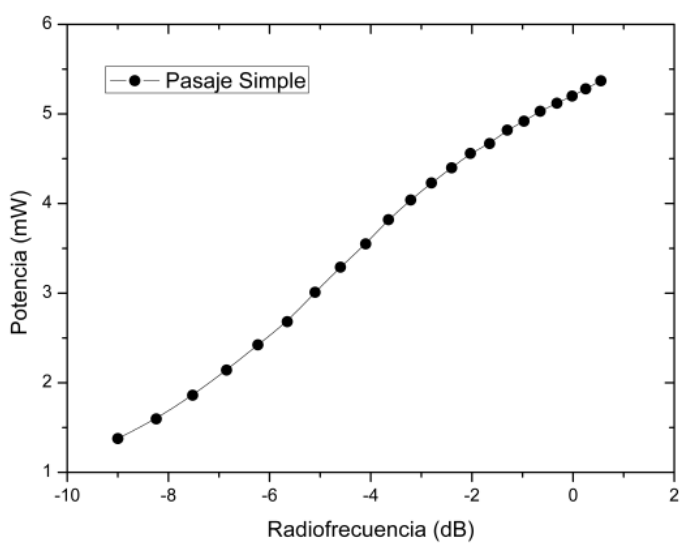

Fig.4. Potencia óptica como función de la frecuencia RF en pasaje simple.

\section{2.b. Configuración de paso doble}

En múltiples aplicaciones, el desplazamiento en el ángulo del haz difractado de orden1 producido al escanear la frecuencia de un láser es un efecto secundario no deseado y puede ser eliminado efectivamente usando el AOM en la configuración de doble paso.

En configuración de doble paso, la salida del orden $m$ del AOM se retro refleja para un segundo paso a través del AOM que conduce a un desplazamiento de frecuencia de $2 m \Delta \omega$ en el haz de doble paso. En esta disposición, cambiar la frecuencia de la onda acústica $(\Delta \omega)$, y por tanto la frecuencia de la luz desviada, no provoca ninguna dirección de la salida de primer orden en la segunda pasada. La salida del segundo paso se contrapropaga con el haz de entrada original. Esto es deseable, ya que permite cambiar la frecuencia sin ninguna orientación del haz de salida, lo cual muestra el problema de cómo separar la trayectoria del haz de salida del haz de entrada. Seguido a esto, una placa de ondas $\lambda / 4$ se coloca justo antes del espejo y hace que la segunda pasada por el AOM se polarice ortogonalmente con respecto al primer pase. Esto permite separar las trayectorias del haz mediante un divisor de haz polarizador. En la Fig. 5, se muestra el set-up del sistema en configuración doble pasaje.

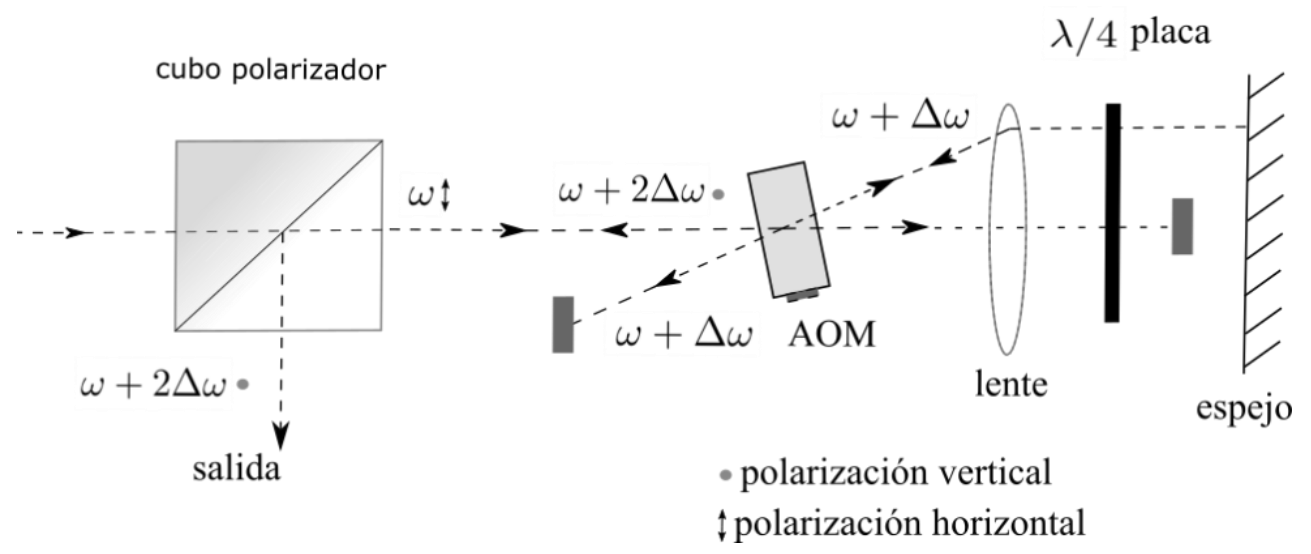

Fig.5. Set-up Experimental para configuración en pasaje doble

Para la alineación del sistema debe tenerse en cuenta una serie de pasos esenciales para obtener la eficiencia máxima de difracción. Lo primero que se debe garantizar es que el haz que pasa por el cubo entre horizontalmente sobre él, para esto deben usarse varias iris a la misma altura para poder obtenerlo, lo cual además facilitará el paso por la lente más adelante. Seguido a esto, se enciende el AOM y se coloca en el foco del haz, menos una pequeña distancia para tener en cuenta el aumento de la longitud del camino óptico dentro del cristal modulador. Este incremento es del orden de milímetros y se puede encontrar en la hoja de datos del dispositivo. Se alinea el AOM para una máxima eficiencia de difracción en el primer orden. Cabe señalar que diferentes AOM tienen diferentes aperturas acústicas activas, esto da 
como resultado que se necesiten cinturas de haz específicas para diferentes AOM y de esta manera lograr eficiencias óptimas de transmisión y difracción [10]. Seguidamente se apaga el AOM e inserte la lente a su distancia focal del AOM (en este caso se usó una lente de $10 \mathrm{~mm}$ para aprovechar de la mejor manera la focalización). Nuevamente, se prueba que el haz esté centrado en la lente para observar que no haya cambios en la posición del haz con y sin la lente en posición. Luego, se inserta una lámina retardador $(\lambda / 4)$ y un espejo de cero grados después de la lente, se centra la lámina en el haz de orden cero y se coloca un iris antes del AOM para evitar la retro reflexión del haz, dejando pasar solo el orden uno de difracción.

Ahora, para obtener la máxima potencia del haz, se colocó un medidor de potencia después del cubo y se ajustó para obtener la máxima potencia usando el espejo y girando el $\lambda / 4$. Los ajustes finales para maximizar la eficiencia de doble paso se pueden realizar utilizando el espejo. Se optimizó la posición de la lente para una deflexión mínima del haz a medida que se cambia la frecuencia AOM. Esto se hace mirando la luz de doble paso a una distancia conocida. La posición óptima para la lente corresponde al punto donde la deflexión del haz es menor.

Después de una óptima alineación del sistema se procede a obtener la eficiencia de la difracción y la variación de la potencia óptica como función de la frecuencia RF. En las Fig. 6 y Fig. 7 se pueden observar las curvas.

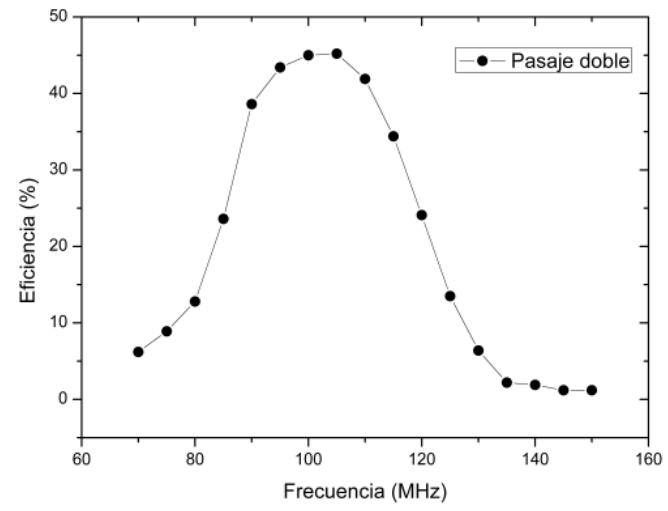

Fig.6. Eficiencia de la difracción como función de la frecuencia en pasaje doble

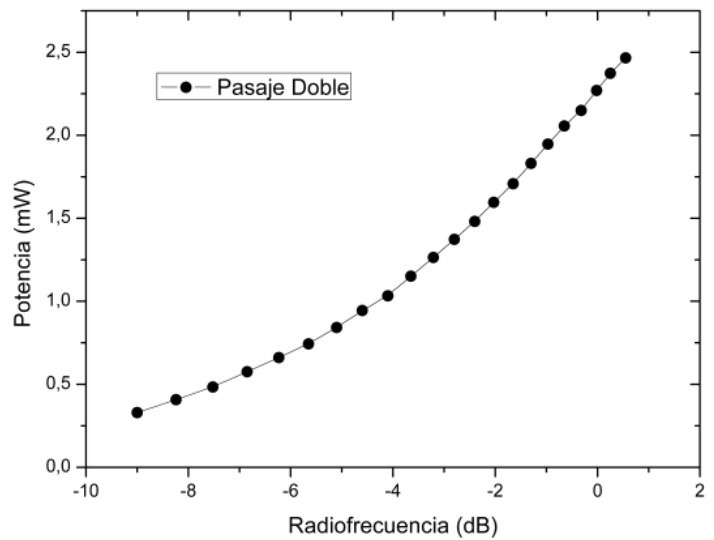

Fig.7. Potencia óptica como función de la frecuencia RF en pasaje doble

Con el AOM correctamente posicionado en la configuración que se muestra en la figura 5, se alineó para obtener la máxima eficiencia de difracción en el primer orden con la frecuencia AOM establecida en $100 \mathrm{MHz}$. Con el AOM apagado, la transmisión del AOM, se midió en 97,2 \%. La eficiencia de doble paso, definida por el cociente del haz de entrada y salida en el sistema de doble paso (entrada en el cubo y salida en el mismo), se midió con la posición de la lente optimizada. Los resultados de estas mediciones se pueden ver en la Figura 6, como la eficiencia aumenta cerca de la 
frecuencia central del modulador y asimismo como disminuye en cuando sigue aumentando, además en la Figura 7 se observa como aumenta la potencia óptica en cuanto aumenta la frecuencia de radiofrecuencia. La Figura 6 muestra una eficiencia máxima de doble paso del 45,2\% a $100 \mathrm{MHz}$. La eficiencia máxima de doble paso es comparable al doble de la eficiencia máxima medida de en pasaje simple $66 \%$.

\section{Comparación paso doble y simple}

El uso de la configuración de doble paso puede eliminar casi en su totalidad los problemas de alineación causados por escanear la frecuencia de un láser con un AOM, en la cual el haz láser viaja a través del AOM dos veces y la desviación del haz se compensa al pasar por segunda vez. La doble modulación de la luz con el AOM significa también que el cambio de frecuencia total es el doble de la frecuencia de modulación. En la Figura 8 y la Figura 9 se tiene la comparación de la eficiencia de la difracción y la potencia óptica como función de la frecuencia RF para las configuraciones de paso simple y paso doble respectivamente.

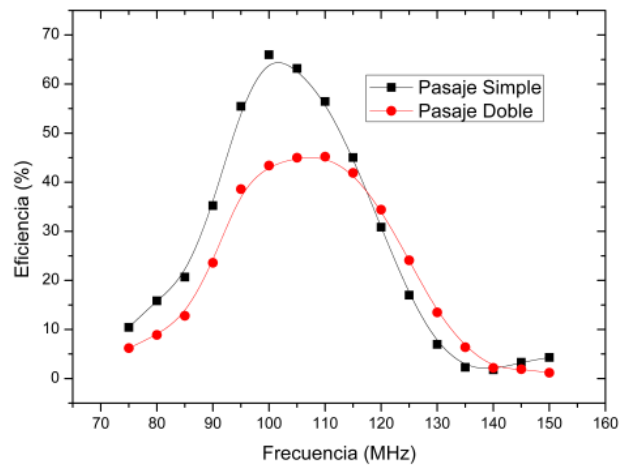

Fig.8. Eficiencia de la difracción como función de la frecuencia en pasaje doble

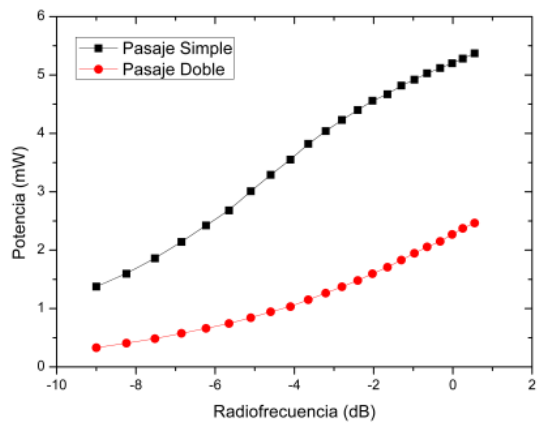

Fig.9. Potencia óptica como función de la frecuencia RF en pasaje doble

Se evidencia en la Fig. 8, como la eficiencia es mayor para el sistema en pasaje simple en comparación con el pasaje doble y en la Fig. 9 como la potencia óptica es mayor en pasaje simple también. Ambos resultados reflejan mayor eficiencia pero sin embargo la estabilidad de la señal dispersada dependerá del ángulo difractado. Además las Figuras 8 y 9 muestran que la eficiencia de difracción, y por tanto la eficiencia de doble paso, de un AOM correctamente alineado, no son constantes cuando se varía la frecuencia del AOM. Estos datos deben recortarse al operar un AOM lejos de su frecuencia central. 


\section{Conclusiones}

En este trabajo se describe una herramienta actualizada para la caracterización e implementación de un sistema de modulación acusto-óptico en configuración de doble paso. Se optimizó el sistema inicialmente en configuración de paso simple, describiendo el montaje experimental y cómo se obtienen las curvas de eficiencia de difracción y potencia óptica como función de la radiofrecuencia. Los resultados obtenidos se resumen en las Figuras 3 y 4, en donde se observaron eficiencia de difracción de $66 \%$ y la relación creciente como muestra de la dependencia de la potencia óptica y la modulación en amplitud en el VCO (Radiofrecuencia). Es plateado que el desplazamiento en el ángulo del haz difractado a primer orden producido al escanear la frecuencia de un láser con un AOM, ésto como resultado de la dependencia del ángulo de difracción del haz y al frecuencia de modulación, y para múltiples aplicaciones es un efecto secundario no deseado el cual puede ser corregido eficazmente usando un sistema de modulación acusto-óptico en la configuración de doble paso. Teniendo en cuenta este planteamiento en la Figura 5 se ilustró el set up experimental y una descripción paso a poso de el montaje óptimo del sistema y así obtener las curvas de eficiencia de la difracción y potencia opticas frente a variaciones en frecuencia encontrando que la eficiencia máxima es de $45,2 \%$ en doble pasaje. Los datos de este trabajo se resumen en la Figura 8, donde se presentan mediciones para las configuraciones. Las curvas de la eficiencia de la difracción y la potencia óptica como función de la frecuencia RF mostrando como en el pasaje simple se puede obtener una mayor eficiencia y potencia óptica pero sin embargo la estabilidad que se puede lograr en el doble pasaje es fundamental, además tal sistema óptico elimina variaciones en la posición del haz y el ángulo durante la sintonización de frecuencia.

\section{Agradecimientos}

Los autores agradencen a Colciecias y la beca doctoral, en la combocatorio 767 (doctorado nacional) del año 2016. 